retention per day. However, when the values are computed it is found that of this alkali retained, 2 c.c. is required for the protein increase, 4 c.c. for the alkali reserve and about 57 c.c., by far the greatest part, for the building of bone.

The effect of acids and alkalis added to the diet has also been studied. When 250 c.c. of $0.1 \mathrm{~N} \mathrm{HCl}$ was given baby $\mathrm{A}$ he retained 67 c.c. of alkali in excess of acid. When 4 grams of sodium bicarbonate, equivalent of 473 c.c. of $0.1 \mathrm{~N}$ alkali was given to baby $\mathrm{B}$ he retained 114 c.c. of base in excess of acid. This is a quantative measure of the effect of acidosis and alkalosis in relation to alkali retention and growth.

It is hoped that studies of this type now being pursued will yield further information on the problems of growth, nutrition and normal and pathological mineral metabolism.

\title{
69 (2029)
}

On the effect of certain drugs, notably quinine, on the acuity of hearing.

By A. G. POHLMAN and F. W. KRANZ.

[From the Department of Anatomy, St. Louis University, St. Louis, Mo., and Wallace Clement Sabin Laboratory of Acoustics, Riverbank, Geneva, Ill.]

The purpose of this investigation was to determine the degree of deafness following the administration of quinine and also to ascertain, if possible, whether or not the tinnitus which accompanies this intoxication has any appreciable effect on the acuity of hearing at any and all pitches. While these experiments were under way the writers found that Macht, Greenberg and Isaacs had published a similar investigation in reference to antipyretics. It was thought desirable, for reasons which will appear later, to check on the results obtained by these writers. The quantitative tests submitted in this paper were all made by $\mathrm{Kranz}^{1}$ with a thermophone provided with a new frequency vari-

1 Kranz, F. W., Phy. Review, 1921, xvii, 384 
ator of his own design. The drug effects were studied both subjectively and objectively by Pohlman ${ }^{2}$. We are indebted to Colonel George Fabyan of Riverbank, Geneva, Ill., for the opportunity of collaborating on this problem of applied physics.

The article by Macht, Greenberg and Isaacs $^{3}$ reviews the literature thoroughly and includes certain notations on the effects of quinine of which we shall speak later. They confined their attention to antipyretics and to certain combinations of these drugs. In a general way, they exhibited small doses of the various drugs to normal individuals and after an hour interval tested the acuity of hearing. This was done by comparing the distance at which a watch tick could be heard before and one hour after the administration of the drug. The difference therefore gave a reading of greater or lesser distance. The authors state that the figures are after all only empirical guides because they recognize "true intensity varies as the square of the distance of the sounding object."

Inasmuch as this paper is mainly directed toward suggesting more definite methods, it may be well to point out certain disadvantages in the use of the watch as a test for minimum audibility. The watch tick is not only a difficult sound, or rather series of sounds, to handle but the conditions under which the usual tests with a watch are taken make quantitative measurements practically valueless for anything approaching accuracy in results. This statement must not be construed as a specific criticism. It is to be lamented that no better quantitative test methods were available for the work on the antipyretics. This same objection holds for the exhaustive study by Kato ${ }^{4}$ on the reflex responses of the $M$. tensor and $M$. stapedius in experimental animals even when the various pitches used were generated by a Galton whistle actuated by a definitely controlled air pressure. The distance at which a given watch tick will be heard is not only dependent on the character of the watch and the manner in which it is held but it is also dependent on the standing wave system in the room in which the test is being conducted. This is never the same in any two rooms or is it the same in any

2 Pohlman, A. G., Annals of Ot., Rhin. and Lary., 1922, xxxi, 1 and 430. 3 Macht, Greenberg and Isaacs, Jr. Phar. and Exp. Med., 1920, xv, 149.

${ }_{4}$ Kato, Arch. and Ges. Physiol., 1913, cl, 569. 
two spots in the same room. The statement of Macht, Greenberg and Isaacs that the intensity of the sound varies as the square of the distance assumes a point source with no reflections from surrounding objects. It is not true for any sort of an enclosed space. For this reason we were interested in the factor of experimental error established for the watch tick tests. It is impossible from the data given to determine the variations in the normal readings because the investigators did not always use the same watch. They were necessarily more interested in ratio diffrences. However one table is offered which indicates the surprising accuracy of the method employed.

The results on single drug effects are summarized in their table 12 on page 163 and which we reproduce as given with one minor correction in the printing, i.e., sodium salicylate should read 74 per cent. instead of 94 per cent.

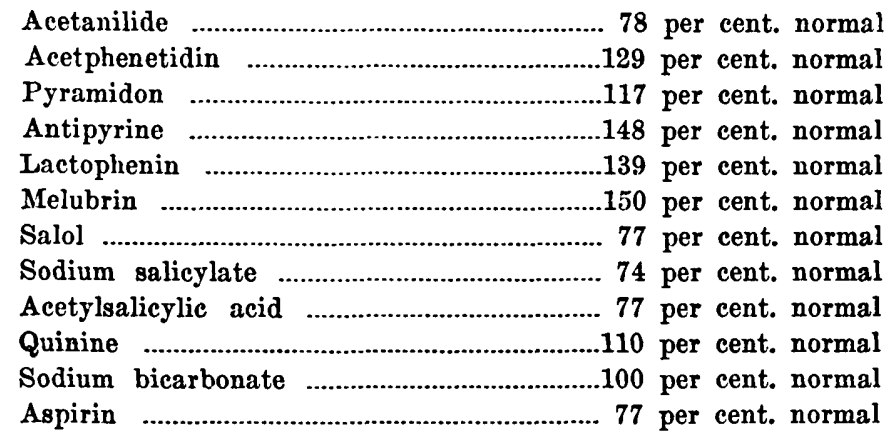

"It was found that acetanilide, sodium salicylate, acetylsalicylic acid, phenyl salicylate, and some other drugs decreased the threshold of hearing." "It was found that acetphenetidin, antipyrine, pyramidon and some other drugs increased the threshold of hearing." The expressions "increased" and "decreased" as applied to the threshold of hearing might in this instance be interpreted in terms of acuity of hearing. The first named group of drugs decreased while the last named group increased the acuity of hearing.

It is extremely important in work on minimum audibility that the variable factor of attention be carefully borne in mind. While no definite information is available on the variable normal for the watch tick tests, the table on the effects of sodium bicarbonate is extremely interesting. In twelve tests in this table the distances before and after administration are exactly the same. 
The range, in the remaining five cases, ran from 6 per cent. minus to 8 per cent. plus. This means that to all intents and purposes seventeen individuals heard the watch at exactly the same distance when the test was repeated in an hour. It would appear, therefore, that Macht, Greenberg and Isaacs had not only overcome the variability in the watch test itself but had also practically eliminated an experimental error in the factor of attention.

The writers determined to check on certain of the antipyretics because it was stated that quinine increased the acuity of hearing. The pitch range of 1000-1800 p.p.s. was used because this particular range was normal for both ears of the subject. Each test was conducted in a similar manner except one (sodium salicylate). The minimum audibility for the scale $1000-1800$ p.p.s. was first determnied and then five grains of the drug was taken in a gelatin capsule. The audibility test was repeated in one hour, and at this time a second dose of ten grains was taken. An audibility test was again made after an interval of another hour. We realize that giving the drug in gelatin capsules has certain disadvantages in the matter of absorption but feel this objection is in part overcome by the second double dose.

No appreciable effect was noted in the case of acetanilide, acetphenetidin and pyramidon. By "no appreciable effect" is meant there did not seem to be a difference which lay beyond the amount of experimental error. However, in testing the remaining drugs much smaller steps in energy variations were employed. The results were obtained in absolute values of intensity for audibility under the various conditions. For the sake of comparison, however, the results are given as ratios; the sound intensity required for audition after taking the drug being given in terms of that required before taking. Thus the percentages greater than 100 per cent. denote a decrease in acuity and the figures less than 100 per cent. signify an increase in acuity. Test I was made one hour after the original dose and Test II one hour later as explained above.

\begin{tabular}{lrrr} 
Drug & \multicolumn{2}{c}{ Single Dose } & \multicolumn{2}{c}{ Double Dose } \\
Salol ......................... Test I. 84 per cent. & Test II. 61 per cent. \\
Aspirin ..................Test I. 124 per cent. & Test II. 124 per cent. \\
Antipyrine ...........Test I. 77 per cent. & Test II. 77 per cent. \\
Sodium salicylate..Test I. $\quad$ (not taken) & Test II. 175 per cent.
\end{tabular}


These results appear to show that aspirin and sodium salicylate decrease the acuity of hearing, while salol and antipyrine increase the acuity. This is a curious result because according to Macht, Greenberg and Isaacs the action of aspirin, sodium salicylate and salol are practically the same; whereas we seem to find an opposite effect in the case of salol. In the twenty-two cases tested with salol they found only one individual who showed an increase in acuity ( 1 per cent.) while in a second test this same individual registered no difference whatever. All others read from a minimum of 53 per cent. to a maximum of 91 per cent. We did not feel that our result was due to an idiosyncrasy on the part of our subject and therefore made a comparison of the absolute intensity values involved in the readings of these four drugs.

This gives us a check which is impossible in the cases reported by Macht, Greenberg and Isaacs. It was found that the sound intensity required to hear the scale of $1000-1800$ p.p.s. when the acuity had apparently been increased by antipyrine was exactly the same as the amount required when the acuity appeared to be decreased by aspirin. Again the increased efficiency under salol required exactly the same amount of energy as the normal taken before the administration of sodium salicylate. This indicated that variation in normal acuity took place on three successive days. It was decided to find out how much variation might take place in a single day. Accordingly a test for normal was taken at 11 A. M. which we called 100 per cent. intensity. The readings of required intensity for audition taken at 3,4 and $5 \mathrm{P}$. M. on the same day without drugs were 88 per cent., 105 per cent. and 135 per cent. respectively. This variation shows a difference of 53 per cent. in two hours and this 53 per cent. covers all of the variations in our figures except that for sodium salicylate.

The results of these experiments are two-fold: first, that these tests, taken under the most favorable experimental conditions, did not yield a difference sufficiently pronounced to be used as a safe basis for deductions on drug effects; and second, all of the variations in amount of intensity lie within a possible error in attention. This same report must be made for morphine sulphate $1 / 2$ gr.; strychnine sulphate $1-20$ gr., and nitroglycerin 1-100th gr. We cannot correlate our findings with the effects as given by Macht, Greenberg and Isaacs. 
It was stated in the beginning of this paper that our chief problem was related to the effects of quinine on acuity of hearing. Witmaak (5) has reviewed the literature on this drug and the following notations are from this source:

Roosa in 1873 reported the evidence of an injection of the drum membrane in three colleagues after ten and fifteen grain doses of the drug. Guder in 1880 experimented on twelve normal individuals and denies a hyperemia of the membrane. Witmak did not find evidence of a congestion in experimental animals and come to the conclusion that quinine has a specific toxic effect on the end organ and cochlear neurones. Ferreri in 1887 reported decrease in both air and bone transmitted sound in a number of cases where large doeses were given (3-4 grams). He found that the acuity of hearing was about as much depressed by 2 grams, as when larger amounts were given. He found also. that the hearing returned to normal in about twenty-four hours. It is unnecessary to devote much space to citing instances which confirm the well-known symptoms of quinine intoxication. As has been said we were more concerned in quantitative tests and in particular the relation of the tinnitus to the decreased acuity of hearing. Inadvertently, however, some interesting observations were made in relation to the toxic effects of a large dose of the drug.

The first step in our tests was the establishment of a curve for minimum audibility as a function of pitch. This was obtained in the following manner: Electrical currents of controllable and measureable frequency and intensity were generated by means of a vacuum tube oscillator and amplifier. The harmonic frequencies were eliminated by the use of suitable electrical filters. The thermophone was used as a sound source. This apparatus takes advantage of the heating effect of the current in a thin platinum strip and the consequent expansion of the adjacent air. The sound intensity produced is calculable in terms of the electrical energy input which can be easily measured. The thermophone unit was mounted in a small telephone receiver case which was held tightly to the observers ear under test. A frequency variator of special design was used so that the observer could at will vary the frequency over the range of nearly an octave. This variation

5 Witmaak, Arch. Ges Physiol., 1903, xcv, 209. 
was a continuous one as distinguished from a variation employing discrete frequencies.

The method of procedure in the determination of the minimum audibility was to vary the frequency back and forth over a given range, the intensity being changed by successive increments. For different intensities near the low limit, different portions of the frequency range will in general be audible, and by a suitable choice of intensity increments, the desired curve of relationship between frequency and necessary intensity for audition may be determined as accurately as is desired. Any peaks or dips in the curve which extend over a narrow range of frequencies, will be easily detected by this method. This would not be possible without a prohibitive amount of time and work if determinations were made with a series of separate distinct frequencies, as is usually done. The intensity was first reduced by the operator until the sound was insufficient to be audible to the subject at any point in the frequency range being used. The intensity was then doubled and the subject, by varying the frequency, determined what portion of the range he could hear. This audible frequency range and the corresponding currents being noted, the intensity was again doubled. This process was continued until all the range was heard. A sufficient number of these frequency ranges, each somewhat less than an octave, were used to cover the frequencies from 320 to 3800 p. p. s.

Results are given in terms of "logarithmic sensitivity," this being the logarithm of the reciprocal of the necessary intensity at the limit of audition, the intensity being expressed in absolute units, ergs per square centimeter per second. Thus the high

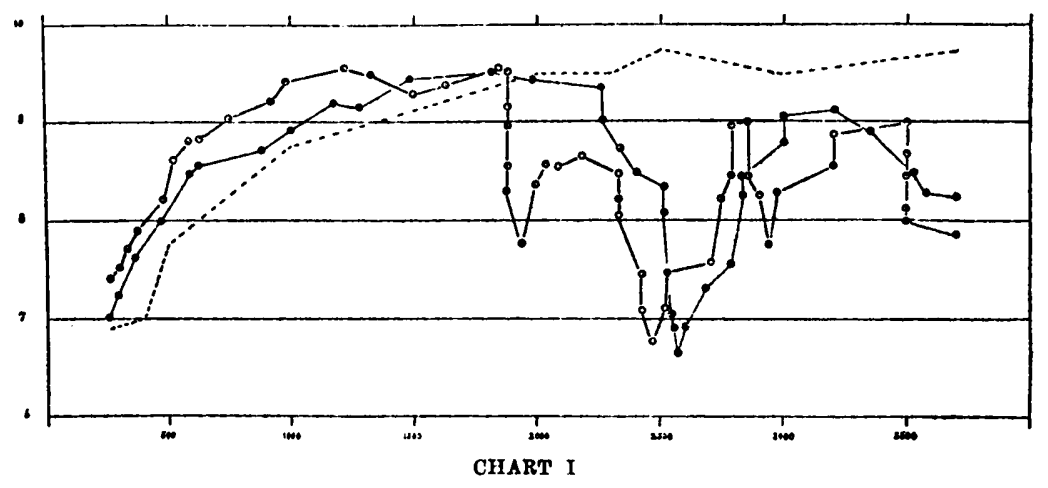


parts of the curve represent the greater sensitivities and on the logarithmic scale equal differences in height have much more nearly equal importance in audition than if plotted on a linear scale. Also small values are not obscured at the bottom of the paper. This logarithmic sensitivity seems to be the most logical way to express sensitivity.

Three curves for minimum audibility are presented in Chart 1. The frequencies in the range tested are indicated on the base line and extend from 256 to 3700 p.p.s. The ordinate quantities are expressed in terms of ergs per square centimeter per second and are plotted on a logarithmic scale from $10^{-6 \text { th }}$ to $10^{-10 \text { th }}$ power. The first curve to be noted is shown by a dotted line and represents the acuity of hearing for the right ear of the subject's fifteen year old daughter taken with the same apparatus. The curve is a normal one and may therefore be used as a guide to the variations from the normal in the two ears of the subject himself. The acuity of the right ear of the subject has been entered as a broken line with light circles while that for the left ear is a continuous line with black circles. The dots on the line indicate the separate frequencies tested and calculated for purposes of the graphic presentation.

The three curves follow a fairly normal picture until the region of 1800 p.p.s. is attained. At no point do the subject's ears differ from that of the control by more than a factor which might be considered beyond a reasonable error in attention. The left ear shows one pronounced spot of decreased acuity (a factor of about 1000 when compared with the control). The lessened acuity begins at 2250 and returns to the normal for this age (43) at 3000 . The right ear, on the contrary, shows three areas of decreased acuity; one pronounced spot at 2450 and two less pronounced ones at 1920 and at 2920 p.p.s. These are separated by two peaks, one at 2200 and another at 2800 . Both ears display a drop-off at the neighborhood of 3400 . (Age change?).

These two curves are interesting because the subject was unaware of any areas of decreased acuity, and even at the present time is not conscious of their presence. The chart gives the reason why the range of 1000-1800 p.p.s. was selected for the tests on antipyretics. If comparisons are to be made of effects on the two ears, this may be done only below the frequency of 1800. The right ear has been chosen for presenting graphically 
the effects of quinine. It was thought essential that the drug be studied in reference to the areas of decreased efficiency to find out if they were depressed as much as the more acute regions. Check reading, however, was also made on the left ear with very similar results.

Test A..-

Twenty five grains of quinine sulphate was taken in five grain gelatin capsules at hourly intervals. The test began soon after the administration of the last dose. The subjective symptoms at this time were: sensation of fullness in the ears and a slight high-pitched tinnitus. Attempts to locate the period of the tinnitus were unsuccessful. It was our intention to follow the tone scale throughout the range for both ears but this was abandoned because the amount of actual decrease in efficiency did not lie beyond a reasonable error in attention.

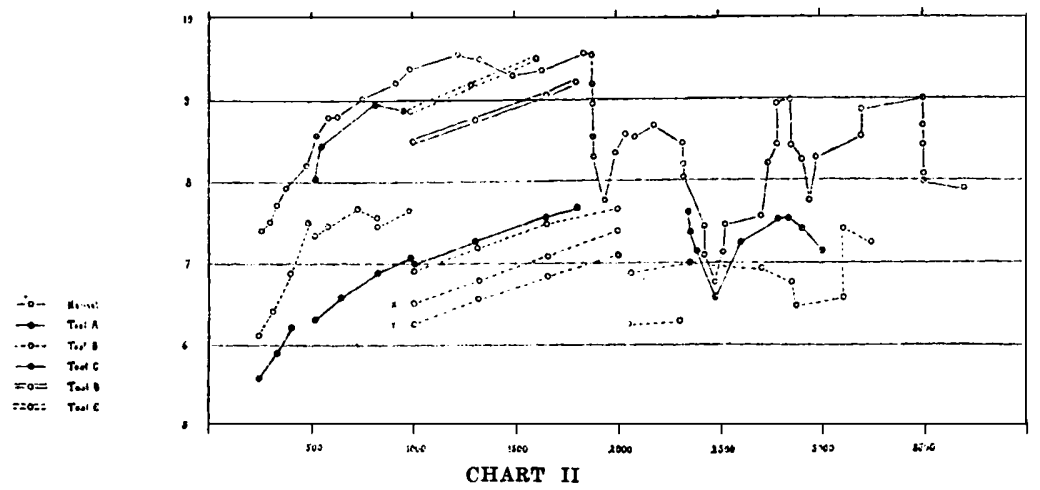

The quantities are plotted in Chart II under Test A. While the decreased acuity area at 2460 was located, the peak at 2800 was not identified. Accordingly, it was determined to exhibit a larger dose of the drug.

Test B.

Forty grains of quinine sulphate were taken in 15 grain doses one half hour apart from 9 A.M. to $12: 30$ P.M. The test began at 3 P.M. Subjective symptons at this time were: a more pronounced tinnitus of the same general character, like the locusts singing in the trees; a more marked sensation of fullness in the ears, and some slight amount of impairment of hearing, particularly for high pitched sounds. This test covered the entire range from 256 to 3250 p.p.s. The results have been entered 
on the chart as Test $B$. While the line for the range $B 1$ goes over fairly well into that of B2, a sharp drop (factor of about 5) was noted in passing to the range B4, where the departure seemed even more pronounced. Two subsequent tests were made over the scale $\mathrm{B} 3$ yielded the variations as shown in the lines $\mathrm{X}$ and $\mathrm{Y}$, (factor of about 10). Accordingly a final test was made from 2100-2300 (B5) when this same sort of decrease in acuity was again evidenced.

As in the twenty five grain experiment the subject missed the peak at 2800 and also failed to identify the decreased acuity area at 2460. It was difficult to interprete these variations and three possibilities suggested themselves. First, the variations might be due to fluctuations in the amount of absorption and excretion of the drug; second, they might be due to errors in attention due to the toxicity of the drug itself in its effect on the nervous system; and third, the pronounced tinnitus might of itself decrease the acuity of hearing.

Test $C$.

Some four days after Test B. the subject took seventy-five grains of quinine sulphate with the intention of pushing the drug to the limit and also eliminate variations in the amount of absorption by spreading the administration of the drug over a longer period of time. Thirty-five grains were taken from $6: 30$ to 9:30 P.M. in seven five grain doses. Forty grains were taken in the morning in ten grain doses hourly from 6:30 to 9:30. The subject did not find his sleep was materially affected and ate a regular breakfast with enjoyment. The test was begun at 10:30 A.M. but had to be abandoned for reasons which will be described more completely elsewhere. The subject became unconscious and was not able to sit up for the test until 4:30 P.M. It was decided to limit the range from $256-1800$ p.p.s and these readings have been entered on the chart under the letter $\mathrm{C}$. The readings of $\mathrm{C} 1, \mathrm{C} 2$ and $\mathrm{C} 3$ line up fairly well.

It appeared from comparing the lower range of Test $B$ with that of Test $C$ that the larger dose influenced the lower part of the range more than it did the upper part, although this is by no means to be accepted as conclusive evidence.

Test D.

This was made on the following morning after a fairly good night's sleep and has been entered under the line marked with 
D. This indicated that the subject had practically returned to normal although a pronounced tinnitus was reported.

Test E.

This made at $4: 30$ the same afternoon is shown which practically coincides with the normal amount of acuity but with the tinnitus about the same as in the morning. The tinnitus gradually disappeared during the next twenty-four hours. Test F.

There still remained the problem of whether the tinnitus itself masked the note or whether it was the toxic effect of the drug which gave rise to the larger than normal variation in passing from one scale to another. It is well known that tinnitus may be produced by an exposure to loud sounds and it was decided to put this to a test. The subject was placed with his head three feet in front of a telephone receiver which emitted an extremely loud note of 1300 p.p.s. This note was sufficiently loud to be heard outside of the building. After three and one-half hours exposure, the subject was immediately subjected to the test over the range from 1000 - 1800 p.p.s. No material alteration was noted in the acuity over the entire range nor was the exact point of the pitch of 1300 influenced. The tinnitus and sensation of feeling of fullness in the ears was as apparent as in the forty grain quinine test and did not entirely disappear for thirty six hours. This seems to show that while the tinnitus appeared subjectively to distract the attention in the test on quinine, it could not in itself be held responsible for the decreased efficiency of hearing.

\section{Conclusions}

We feel the following conclusions may be drawn from these experiments on quinine:

First. A twenty five grain dose did not materially influence the acuity of hearing.

Sccond. A forty and a seventy five grain dose produced a pronounced decrease in acuity. The larger dose apparently affected the lower part of the scale (256 to 1000) more markedly than the upper part $(1000-1800)$. We are, of course, not sure of the amount of drug absorbed and will gladly turn over the subcutaneous or intravenous injection to someone else. There can be no question that the drug was not pushed to the limit in Test $C$. 
Third. The amount of actual loss in acuity of hearing was not proportionate to the loudness of the tinnitus. After hearing returned to normal the tinnitus continued for at least twentyfour hours.

Fourth. A tinnitus produced by a continued loud sound gives rise to similar sensations of fullness in the ears and does not bear any definite pitch relation to the frequency of the sound causing the condition. The acuity of hearing was not impaired after three and one half hours exposure.

Fifth. Recovery of normal hearing after ingestion of quinine occurs in 24 to 36 hours.

Sixth. The toxic affect of quinine influence not only the auditory sense, but the entire nervous apparatus is affected so that definite readings with larger doses are not possible.

Seventh. We present herewith a study in the application of modern methods in the measurement of acuity of hearing. The disadvantages shown in this accurate method practically rule out the watch tick tick and the tuning for $\mathrm{K}$ tests in their application to minimum audibility.

\section{$70(2030)$}

Studies on lung volume. IV. Investigations on admixture of air in the lungs with other air.

By CHRISTEN LUNDSGAARD and KNUD SCHIERBECK.

[From the Medical Clinic of the University of Copenhagen, Denmark.]

Several methods of importance for the study of the physiology and pathology of respiration and circulation require (1) that it is possible to produce full admixture of air within the lungs with other air and (2) that the exact conditions necessary for full mixture can be ascertained in a given case. This and the following paper is a short report of experiments dealing with questions.

Technique. 3 liters of oxygen (Allen-Pepys' method) or of hydrogen plus oxygen (Davy-Durig's method) are introduced in 